\title{
La traducción, ¿un género en sí mismo?
}

\author{
Translation, a genre in itself?
}

\author{
Ana María Gentile \\ Universidad Nacional de La Plata, Argentina \\ agentile@fahce.unlp.edu.ar
}

\section{Resumen}

Tanto desde el punto de vista investigativo como institucional, la traducción como objeto de estudio se encuentra en la encrucijada de numerosas disciplinas. Sin embargo, su estatus arrastra el peso de su condición ancilar. Frente a las nuevas reflexiones que parten de las ideas deconstructivistas, por un lado, e intersemióticas por otro, las cuales coinciden en señalar la ausencia del texto original, nos proponemos contribuir, desde el análisis del discurso, a la definición de la traducción como un género en sí mismo. Para ello, retomaremos nociones básicas como ethos y enunciación, entre otras, con el objeto de indagar la factibilidad de tal empresa.

Palabras clave: traducción, género discursivo, enunciación, texto, ethos del traductor

\begin{abstract}
Translation, as an object of study, is at the crossroads of numerous disciplines, both from the research and institutional points of view. However, its statute carries the weight of its ancillary condition. In face of the new reflections that start with deconstructivist ideas, on the one hand, and intersemiotic ideas on the other, which point out the absence of the original text, we propose to contribute, through discourse analysis, to the definition of translation as a genre in itself. To achieve this, we will return to basic notions such as ethos and enunciation, among others, in order to delve into the feasibility of such undertaking.
\end{abstract}

Keywords: translation, discourse genre, enunciation, text, translator's ethos

Fecha de recepción: 22 de enero de 2020 | Fecha de aceptación: 6 de agosto de 2020

Cómo citar este artículo (MLA): Gentile, Ana María. “La traducción, ¿ Un género en sí mismo? Estudios del Discurso 6.2 (2020): 48-67. 
La traducción, ¿un género en sí mismo?

\section{Introducción}

a disciplina traductológica, o lo que se ha dado en llamar estudios de trade indagación. Este interés por la traducción no se manifiesta sólo desde la lingüística o desde la literatura, sino que convoca a análisis y reflexiones en diversos campos como la sociología, la literatura comparada o la filosofía, por citar solo un puñado de disciplinas. Así pues, las teorías de corte eminentemente prescriptivo que reivindicaban la traducción como una operación entre lenguas se vieron confrontadas en las últimas décadas a la aparición de perspectivas más descriptivas y con vocación explicativa frente a los problemas que el hecho traductor plantea. De este modo, distintos enfoques provenientes del análisis del discurso, de la pragmática, de la teoría comunicativa, entre otros, fueron enriqueciendo las reflexiones en torno a la traducción, más allá de la comparación de fenómenos sintácticos, léxicos o morfológicos. Entre esos otros enfoques, los estudios culturales contribuyeron a crear nuevos interrogantes y herramientas de descripción y explicación del fenómeno de la traducción de la cultura, de la mano de teorías como la del Polisistema (Even-Zohar, La posición y Toury, Descriptive Translation Studies) o la Sociocrítica de la traducción (Brisset, Sociocritique de la traduction).

Se ha hecho referencia a la traducción como actividad, como proceso, como acto comunicativo, como resultado, como práctica intercultural, sin embargo, ha habido pocas reflexiones sobre la traducción como género en sí mismo. Por ello nuestro recorrido pretende contribuir a esta indagación desde las teorías provenientes principalmente del análisis del discurso y de la enunciación. El presente trabajo parte de nuestra experiencia de formación durante la cual surgieron los primeros interrogantes y las exploraciones que luego se erigieron en hipótesis para nuestras investigaciones. En una segunda instancia, nos detenemos en las teorías y enfoques que a nuestro entender indagan la particularidad de la traducción por fuera de los paradigmas tradicionales de la equivalencia y de la comparación de lenguas. Finalmente, escogemos los argumentos más representativos y pertinentes desde el Análisis del Discurso y de las teorías enunciativas que nos permiten sostener la caracterización de la traducción como un género en sí mismo. Dado que 
se trata de nuestras primeras indagaciones en este tema, dejamos varios frentes abiertos para continuar profundizando una problemática actual, interesante desde varios enfoques y particularmente atractiva para la investigación y la formación de traductores.

\section{La traducción: de una enunciación ignorada...}

En nuestra formación en la carrera de Letras, completada luego de terminar estudios de traducción, observábamos la ausencia de una problematización, reflexión, o siquiera una mención, de la traducción como una enunciación mediada en las obras literarias. Las lecturas en lenguas extranjeras se efectuaban en su versión castellana y a lo sumo se aconsejaban determinadas ediciones, pero se ignoraba, quizás sobreentendiéndola, la dimensión de la traducción, su importancia como instancia mediadora, así como la intervención del traductor y el funcionamiento del texto en la cultura receptora.

Recuerdo plantear esta cuestión en más de una oportunidad a los docentes, cuestión que era crucial, a mi entender, en la interpretación del hecho literario, de la obra y de su contexto, de la red de significaciones en juego y por ende de la crítica y análisis literario, pero en los ochenta en Argentina todavía prevalecía un paradigma lingüístico y estructuralista; esta realidad fue paulatinamente modificada a partir de la introducción de nuevas asignaturas de corte más discursivo y cultural. Las obras se analizaban como si fueran originales y la intervención del traductor carecía, salvo pocas excepciones de docentes provenientes de una formación en lengua extranjera, de una reflexión consciente y realista.

Ahora bien, desde el momento en que la traducción deja de ser considerada como una operación de transferencia de una lengua a otra para pasar a plantear una relación con un texto y con una enunciación preexistentes, numerosas son las cuestiones que atraen hoy la mirada del investigador, particularmente, desde el Análisis del Discurso y los fenómenos de la enunciación. Ilustrativa de este giro es la perspectiva de Jean-Yves Masson al inaugurar el Primer Congreso Mundial de Traductología organizado por la Société Française de Traductologie que, 
La traducción, ¿un género en sí mismo?

en memoria de Umberto Eco, se celebró en abril de 2017 en la Universidad de Nanterre, París. En su alocución de bienvenida, Masson enfatizó la fuerte desvalorización de la actividad de los traductores a lo largo de la historia, hecho que, sostuvo, llevó a desconocer durante mucho tiempo la dimensión enunciativa, creativa, social e ideológica del acto traductivo como práctica discursiva.

Desde el punto de vista teórico, es de destacar que la traductología se ha nutrido de los nuevos avances de disciplinas afines, y en este caso son los desarrollos de la lingüística discursiva, del análisis del discurso y de las teorías de la enunciación los que han alimentado la idea, junto con el paradigma deconstructivista, de una autonomía del texto traducido respecto de su original. Por un lado, la perspectiva discursiva y enunciativa lo efectuó a partir de la idea de que la traducción se acerca ya sea al discurso referido, a la reformulación o a la re-enunciación; el enfoque deconstructivo hizo lo propio sobre la base de la inexistencia de un texto original y la indeterminación de una voz que hasta hace no mucho continuaba sujeta a la figura del autor tributaria del Romanticismo.

\section{... a una presencia visibilizada}

Hoy en día podemos preguntarnos: ¿A quién se le ocurriría hoy afirmar que el cuento de Caperucita Roja es una traducción? Los ejemplos se pierden en la noche de los tiempos y merecen análisis pormenorizados, pero creemos que este cuestionamiento nos lleva a tomar consciencia de un fenómeno que, a la manera de la carta robada, es tan evidente que pasa inadvertido.

Desde la dicotomía entre textos religiosos y textos literarios, que San Jerónimo utilizó para convalidar dos distintas maneras de traducir, la literal y aquella en la cual el sentido es prioritario, respectivamente, la historia de la traducción ha oscilado entre dos polos que Schleiermacher (Sobre los diferentes métodos de traducir) sintetizó de la siguiente manera: el traductor tiene que tomar la decisión de acercar al autor hacia el lector o bien acercar al lector hacia el autor. La primera decisión es la que caracterizó al movimiento de las "bellas infieles" hasta el límite de adaptar según el gusto de la época toda obra clásica. La segunda orientó las 
reflexiones de muchos escritores como Walter Benjamin, (La tarea del traductor) Ortega y Gasset (Miseria y esplendor de la traducción) y Antoine Berman. (Lépreuve de létranger). En este recorrido, la omnipresencia del texto original y la referencia constante a éste marcaron una historia que comenzó a cambiar con las teorías deconstructivistas. En efecto, en su propósito de echar por tierra las oposiciones binarias, Derrida (Le monolinguisme de l'autre) sostiene que no hay un original y una traducción, ya que todo original remite por esencia a otro. Este indeterminismo es la base de una conceptualización teórica en la que el carácter ancilar de la traducción desaparece, y con éste no hay supremacía del original sobre su traducción ni tampoco un significado estable que remita a la lengua pura a la que hacía referencia Benjamin.

Sin embargo, el estatus invisible de la traducción no fue siempre así. Ballard (De Cicéron à Benjamin) señala que la historia de la traducción es la gran ausente en los textos más emblemáticos de la teoría traductológica. Esta afirmación, realizada hace casi treinta años, mantiene cierto viso de verdad aunque está siendo superada, al menos en Francia a nuestro saber, por una serie de publicaciones sobre justamente la historia de la traducción en ese país. En este sentido también trabajan diversos grupos como el colectivo Histal de Canadá (http://www.histal.net/) dirigido por el Dr. Georges Bastin, y tesis individuales que contribuyen a trazar la historia de la traducción en nuestro continente. En el espacio de este trabajo, sin entrar en casos particulares, nos limitamos a señalar dos hitos históricos que pueden ayudarnos a trazar un recorrido de la traducción como género.

A riesgo de ignorar muchísimos datos, destaquemos dos que han despertado nuestra atención en el marco de la reflexión que nos ocupa. En primer lugar, el papel que cumple Roma en su deseo de anexión a la cultura griega: en lo que se conoce como el primer escrito teórico sobre la traducción, su autor, Cicerón, se refiere a la importancia de la traducción como molde de un género, la imitación, reflexión que da lugar en la teoría traductológica a la noción de equivalencia, tan polémica y criticada, pero a su vez tan rica para matizar y relativizar. En segundo lugar, el lugar que ocupa la traducción en la Francia del siglo XVII, período en el que el movimiento de las "bellas infieles" contribuyó a que la traducción fuera considerada un género literario propiamente dicho. Las traducciones "libres" se 
La traducción, ¿un género en sí mismo?

alejaban del original y se adaptaban al gusto de la época, en una especie de hiperadaptación o, para emplear un término actual, de localización a ultranza.

En el siglo xx, dos pilares abonan la caracterización de la traducción como género autónomo desde posiciones diferentes; nos referimos a las reflexiones de Ortega y Gasset y a la Teoría del Polisistema. Deténgamos en ellas.

\section{La traducción como género autónomo: de Ortega y Gasset} a la Teoría del Polisistema

En nuestras búsquedas e indagaciones, hemos encontrado una referencia interesante para nuestro enfoque por dos motivos: por la actualidad de su pensamiento y por provenir del campo hispanohablante. Nos referimos a las reflexiones esbozadas por el filósofo español José Ortega y Gasset en su ensayo pionero "Miseria y esplendor de la traducción" de $1937 .{ }^{1}$ Efectivamente, en este trabajo Ortega y Gasset afirma, teniendo como base la hermenéutica alemana y más específicamente lo sostenido por Schleiermacher, que la traducción no es un doble del original, ni siquiera comparte con el original su mismo género, ya que contiene sus propias normas y su propio objetivo. Así, la traducción no es ni una transustanciación ni la obra en cuestión, sino un camino de acceso a ella. La vigencia de este pensamiento supera el enfoque hermenéutico y nutre las reflexiones actuales en torno de la problemática de la traducción de las ciencias humanas, en especial los casos de intraducibilidad (Cassin, Vocabulaire européen des philosophies) y de los falsos amigos conceptuales. La tarea del traductor se visibiliza en notas al pie, prefacios, prólogos, glosarios, que buscan reconstruir el sentido del original frente al desafío de la pérdida muchas veces inevitable.

Más cercana a nuestro presente, sin lugar a dudas fue la Teoría del Polisistema la que mejor señaló el carácter autónomo de la traducción. Esto es así dado que,

${ }^{1} \mathrm{Si}$ bien la primera edición de este ensayo se desplegó en sucesivas entregas del diario La Nación de la República Argentina, remitimos al lector a la edición de las Obras Completas de 1983 que figuran en la bibliografía. 
según este enfoque, la traducción no es una mera reproducción de su original, sino que gana un espacio y ocupa un lugar en la cultura receptora. Por lo tanto, más que hablar de la fidelidad o no de la traducción a su original, los detentores de este enfoque se preocupan por indagar acerca del funcionamiento diferencial que tiene la traducción en la cultura de destino. Esta perspectiva ha significado una revolución en los conceptos básicos de la traductología como son la equivalencia (ya no estable sino dinámica y cambiante), la función (no del texto sino de la traducción como un fenómeno que impacta en la cultura receptora y que interactúa de manera diferente en un sistema diferente al del original) y las normas (según Toury, la traducción está regida por sus propias normas preliminares y operativas que nada tienen que ver con la norma prescriptiva). De este modo, en los Descriptive Translation Studies subyace la idea de que las traducciones son textos de pleno derecho y no meras representaciones de un texto previo (Toury), por lo cual el vasto proceso de creación, de reelaboración escrituraria, profundamente situada, discursiva y cultural, gana terreno frente a la simple derivación.

Esta reivindicación de la traducción también conlleva la indagación acerca de sus características sistemáticas, no ya de casos individuales sino de universales, como las investigaciones de los Corpus-based Translation Studies (CTs) de traducción (Baker) o las leyes (Toury), entendidas éstas más bien como regularidades dada la imposibilidad de concebir la traducción dentro de un paradigma naturalista. Si bien es tarea compleja descubrir cuáles son los tan mentados universales de traducción, existen estudios serios y detallados dentro de esta perspectiva. Los mismos, mediante la comparación de textos escritos por nativos, se caracterizan por una tendencia a la normalización, a la explicitación y a la simplificación. No obstante, la búsqueda de universales resulta engorrosa dada la dimensión histórica que inevitablemente atraviesa todo gesto traductor. De allí que, ya en un plano más sociológico, pero respaldando esta perspectiva de la autonomía de la traducción, el estudio de casos sobre el teatro en Quebec realizado por Brisset da cuenta de las bases socio-discursivas de la operación traductora. Similar enfoque adopta Willson en sus análisis de las traducciones realizadas por la revista Sur entre las décadas de 1930 y 1960 en la Argentina.

La problemática de la obra traducida genera igualmente otras caracterizaciones de distintos teóricos. Así, Risterucci-Roudnicky sostiene que existe en la obra 
traducida una dualidad irreductible e intrínseca producto de tres tipos de operaciones: la transposición lingüística, la transferencia cultural y la reapropiación extranjera. Esta hibridez textual es un rasgo característico que atañe tanto a la coexistencia de autor y traductor como a los indicios de la presencia extranjera en el texto traducido. Dichos indicios pueden ser tanto de índole tipográfica (comillas, cursivas), lingüística (palabras extranjeras), cultural (los realia, la onomástica) como intertextual (citas, alusiones a otras lenguas, culturas y literaturas diferentes del receptor y a veces del autor).

Como dijimos anteriormente, todos estos estudios y muchos otros que exceden el espacio del presente trabajo, han hecho referencia a la traducción como actividad, como proceso, como acto comunicativo, como resultado, como práctica intercultural... ¿será posible referirse a la traducción como género?

\section{La noción de género discursivo}

Los géneros textuales han sido objeto del análisis traductológico dentro de lo que Pym ha dado en llamar el paradigma de la finalidad, abordaje funcionalista que, a pesar de sus idas y vueltas entre texto de partida y texto de llegada (Reiss con su idea de que el traductor debe mantener la función del texto de partida, Vermeer con su énfasis en el texto de llegada) han sumado voces en la visibilización de la figura del traductor, superando el paradigma de la equivalencia lingüística.

Las numerosas descripciones de las obras traducidas nos invitan, ya desde el plano textual, a definir un conjunto de indicios - no exhaustivos- que ameritan la calificación de la traducción como un género en sí mismo. Aquí, una vuelta por la noción y sus complejidades se impone.

Recordemos que el mentor del concepto de género discursivo ha sido Mijaíl Bajtín² quien, a partir del estudio de la obra literaria de Dostoievsky, introduce las

\footnotetext{
2 Si bien Bajtín desarrolló sus teorías a partir de los años veinte, remitimos al lector a la edición de 1982 que figura en la bibliografía y que consigna su autoría (a diferencia de otras obras donde figura con el nombre de Voloshinov).
} 
nociones de polifonía y analiza la dimensión interactiva del lenguaje. En el caso de los géneros en la perspectiva bajtiniana, todas las esferas de la actividad humana están relacionadas con el uso de la lengua, la cual tiene lugar a través de enunciados concretos pertenecientes a los participantes de estas esferas. Estos enunciados reflejan las condiciones específicas y el objeto de cada una de las esferas por su contenido temático y por su estilo verbal y, además, por su composición o estructuración. Cada una de estas esferas de la actividad humana tiene su propio estilo de comunicación, es decir, su género discursivo. Los géneros son determinados por funciones (científica, técnica, periodística, etc.) y condiciones específicas para cada esfera de la comunicación discursiva. La diversidad de géneros es muy extensa y por ello se debe diferenciar entre géneros discursivos primarios (simples) y secundarios (complejos).

Para Bajtín, el enunciado es la unidad real de la comunicación discursiva. Así, entiende los géneros discursivos como enunciados estables elaborados por cada esfera del uso de la lengua. Hoy en día, la noción de género abarca no solo aquellos productos culturales basados en la predominancia de la palabra sino todos aquellos generados por las nuevas tecnologías y los medios de comunicación.

Como bien indican Charaudeau y Maingueneau, la noción es problemática y se define generalmente alrededor de dos perspectivas: o bien se habla de género de texto, pensando en sus características de composición textual, o bien se habla de género de discurso, aludiendo a sus condiciones de producción.

Dentro de los parámetros para ser considerados indicios de géneros discursivos particulares se encuentran las finalidades, los actores, los temas propios de ese ámbito y las formas verbales y no verbales propias y adecuadas para cada caso. Sin ánimo de agotar el análisis, sí nos interesa detenernos en la figura del traductor como instancia mediadora de enunciación de su voz, ya que consideramos que, desde esta perspectiva, los estudios recién comienzan, como veremos a continuación en lo que atañe a la noción de ethos. 
La traducción, ¿un género en sí mismo?

\section{El ethos, noción discursiva y traductológica}

Proveniente de la Retórica de Aristóteles, la noción de ethos convoca actualmente numerosas investigaciones desde variadas perspectivas pragmáticas, sociológicas, y enunciativas, entre otras. A pesar de ser considerado un "concepto heurístico" (Woerther), es decir un concepto abierto a la interpretación, el ethos ha sido uno de los redescubrimientos de la lingüística discursiva, de la nueva retórica, de la teoría polifónica y de los desarrollos de la pragmática. Definida generalmente por la retórica clásica como la imagen de sí mismo que da un orador por su manera de hablar, la noción fue reformulada por Ducrot y por Maingueneau (Genèses $d u$ discours), y se extendió al campo de la literatura, de la traducción, de las lenguas extranjeras desde variadas perspectivas como la pragmática, la teoría de la enunciación y las indagaciones sobre el multilingüismo y la interculturalidad.

Maingueneau (Ethos, scénographie, incorporation 48) la retoma como un parámetro más del discurso sobre esta observación de partida: “[...] todo discurso, oral o escrito, supone un ethos: implica cierta representación del cuerpo de su garante, del enunciador que asume su responsabilidad. [...] el ethos no debe aislarse de los otros parámetros del discurso; contribuye de manera decisiva a su legitimación". El mismo autor sostiene más tarde (Maingueneau, Ethos, scénographie, incorporation 87), asimismo, que "[...] toda habla viene de un enunciador encarnado; incluso escrito, un texto es sostenido por una voz, la de un sujeto más allá de un texto".

A partir de este redescubrimiento, el ethos se torna un concepto interesante para los estudios traductológicos, en tanto interviene activamente en el terreno del diálogo y de los intercambios culturales. Como ya hemos mencionado, el giro cultural y sociológico de las últimas décadas destierra la imagen de la traducción como un texto menor, ancilar, subordinado a un original estable y único. De este modo, sabemos que la traducción es más que el proceso de transferencia lingüística en el que la figura del agente (el traductor, claro está) es invisibilizada: es, antes bien, una instancia de re-enunciación, una transmisión del conjunto de la situación de enunciación de partida. Así, el responsable de la traducción posee su propia voz, la cual no solo se localiza sino que se caracteriza. 
Dicha dimensión enunciativa, ignorada por la idea de la lengua como un sistema estático solamente abordado desde el paradigma traductológico de la equivalencia, es revalorizada hoy como práctica discursiva situada y, por lo tanto, creativa, ideológica y social. En este sentido, la noción de ethos en el campo de la traductología es objeto de variadas indagaciones no solo en las lenguas inglesa y francesa, sino también en lengua española. Prueba de ello son las investigaciones más recientes de Spoturno (The Presence and Image, El retrabajo del ethos) y Ghirimoldi (La lectura de textos traducidos, Retos de la traducción), así como proyectos de investigación acreditados por la Universidad Nacional de La Plata, Argentina, en la cual desarrollo proyectos.

Una de las teorías que complementan el marco teórico del estudio del ethos es el análisis de la argumentación de Ruth Amossy (Images de soi dans, Largumentation dans le discours, Argumentation et Analyse, La présentation de soi), quien postula que todos los discursos están atravesados por la argumentación ya que buscan producir como mínimo un efecto en el destinatario. Recordando a Benveniste (Problèmes de linguistique générale), para quien la definición de discurso comprende toda enunciación con un locutor cuya intención es influir de alguna manera en el otro, podemos observar que la problemática de la argumentación según esta perspectiva es consistente con las ideas que nutren la denominada Escuela de la Manipulación (Hermans, The Manipulation of Literature), para la cual traducir nunca es un acto neutral, sino una operación discursiva de naturaleza ideológica y política.

En contacto directo con la noción de ethos, el caso de la re-enunciación empalma la figura del traductor con la caracterización de la categoría de mediación que implica toda traducción, como veremos a continuación.

\section{La traducción como operación de re-enunciación}

El término mismo de traducción encierra una metáfora espacial. Así, la idea de trasladar, de ir de una lengua de partida a una lengua de llegada, de comprender 
La traducción, ¿un género en sí mismo?

un texto fuente para re-expresarlo en un texto meta, implica una direccionalidad que forma parte ya de nuestras formas de referirnos al hecho traductor. Sin embargo, la idea de re-enunciación desplaza la metáfora espacial hacia otra inscripta en un "aquí y ahora" situado, ya que:

Si se acepta considerar el texto literario como el producto de un acto de enunciación, se considerará lógicamente su traducción como un acto de re-enunciación. Esta propuesta es muy simple pero escasamente explorada, sin duda porque exige desmitificar la invisibilidad del traductor». (Suchet, La traduction 32, nuestra traducción).

Esta noción, pues, nos ayuda a caracterizar la traducción como un género en sí mismo, de la misma manera en que el análisis del sistema literario y cultural que recibe al texto traducido contribuye a entender las decisiones, estrategias y contextos del mismo, dando lugar así a un texto diferente del original y, por ende, insistimos, susceptible de ser caracterizado como un género autónomo. Vale quizás una aclaración: cuando decimos autónomo no pretendemos escindirlo de su relación con un original (par indisociable si evocamos la idea benjaminiana de la traducción), aunque sí destacar su naturaleza de diferente respecto de su texto previo. Tal noción diferencial es la que sostiene Suchet al definir el ethos del traductor como la "imagen de la distancia constitutiva de toda enunciación tal como es negociada entre el texto original y su traducción" (Suchet, La traduction 409, nuestra traducción).

Suchet se refiere al texto literario, pero entendemos que tal propuesta puede aplicarse cabalmente a todo tipo de textos, tanto escritos como orales. El caso de la traducción científica, técnica e incluso jurada (o pública, como se la denomina en Argentina), sin olvidar las situaciones de interpretación simultánea o consecutiva, también merecen que nos detengamos en reflexiones desde este punto de vista (Gentile, Los textos jurídicos).

En este marco, la definición de la traducción como "una operación de re-enunciación por la cual un enunciador se sustituye a una instancia de enunciación anterior 
para hablar o escribir en su nombre en una lengua considerada diferente" (Suchet, L'Imaginaire hétérolingue 28, nuestra traducción), encuentra todo su sentido.

La idea de la lengua considerada diferente resulta también relevante para nuestra propuesta ya que se acerca a la noción de heterolingüismo, es decir "la presencia en un texto de giros extranjeros, bajo cualquiera de sus formas, así como de variedades (sociales, regionales o cronológicas) de la lengua principal" (Grutman, Des langues qui résonnent 37 , nuestra traducción).

Considerar la traducción como un caso particular de heterolingüismo es introducir la figura del traductor y su ethos como una instancia de re-enunciación en el texto traducido, con todo lo que ello implica. De este modo, la traducción se encuentra legitimada ya que la distancia que la separa de la enunciación original o primera es constitutiva de esta enunciación. La intervención más o menos pronunciada del traductor es la clave para analizar la negociación que se produce entre el original y su traducción. Por ejemplo, Suchet señala que las dislocaciones enunciativas explícitas, en particular las notas a pie de página, delimitan los territorios del "sí mismo" y del "otro". Por el contrario, la ausencia de marcas indica la imposibilidad de adoptar una instancia de enunciación y prioriza la indistinción, momento en el cual el traductor se invisibiliza como mediador. El traductor como enunciador puede resultar interesado en actuar como una instancia lingüística y cultural o bien abandonar al lector frente a ciertos enigmas e incógnitas. De este modo, la ausencia de explicación produce un efecto de encriptación voluntaria, mientras que la glosa indica que el responsable se esfuerza por servir de guía o de mediador intercultural. Asimismo, la presencia de préstamos, especies de islotes que marcan la presencia del otro en el discurso, en este caso la de la lengua extranjera, por medio de convenciones tipográficas como las comillas o las cursivas, revelan la inserción de la voz extranjera en el territorio del texto traducido. Explícita en el paratexto y en los mecanismos de préstamo, esta presencia es insoslayable y funciona dentro de un texto muy diferente a su original.

Tomemos dos casos de la literatura para ilustrar estas herramientas teórico-metodológicas. Para ello, vamos a referirnos a la traducción al francés de la obra de Julio Cortázar Rayuela que se publicó en 1963, y que con el título Marelle se publica en 1966 bajo la pluma de las traductoras Laure Guille-Bataillon y Françoise Rosset. 
El primer caso lo ejemplifica la lectura de la página 193 del original: “... Para colmo tenía ganas de matear y se le había acabado la yerba: es decir, le quedaba yerba para medio mate [...] Con clavos derechos y yerba la siesta sería más tolerable." En la página 260 de Marelle, el lector francés va a leer: «... Sans compter qu'il avait envie de se taper un maté et qu'il n'avait plus d' 'herbe', ou plus exactement il ne lui en restait que pour un demi-maté[...] Avec des clous bien droits et du maté, la sieste serait plus tolérable. » Según el enfoque de las heterogeneidades enunciativas de Authier-Revuz (1984), la heterogeneidad marcada por las comillas destaca, a manera de islote en el territorio del texto, el elemento buscado. En este caso, la yerba mate característica de la infusión popular en Argentina sobresale mediante las comillas en una intervención traductora ante la cual nos podemos preguntar: ¿qué idea de la lengua pudo haber tenido la traductora?, ¿qué concepción del término propio de la cultura extranjera motiva la elección de las comillas? o, lo que quizás puede tener una respuesta más concreta, ¿qué lee e interpreta el lector? Una pequeña encuesta llevada a cabo entre lectores franceses arrojó como resultado una percepción de ese elemento como la "hierba", es decir la droga, justamente por la presencia de las comillas. Lejos de realizar este cotejo entre original y traducción como una muestra de errores, dificultades e inequivalencias, lo que nos interesa indagar aquí es la re-enunciación traductora en cuanto ésta es reveladora de posicionamientos a la hora de asumir la responsabilidad de la enunciación, acto que implica un ethos del traductor determinado. En este caso, la traductora asume la alteridad de la referencia cultural y marca dicha heterogeneidad negociando la distancia enunciativa que existe entre el original y su escritura.

El segundo ejemplo que podemos mencionar es la frase que aparece en la página 187 de Rayuela: "En la parrilla, Oliveira empezó a tomar vino y a comer chorizos y chinchulines", re-enunciado en la página 251 de Marelle como: "Une fois au bistrot, Oliveira se mit à boire du vin rouge et à manger du chorizo et des chinchulines, autrement dit des tripes en tresse." La traductora ha asumido que la referencia cultural (por supuesto no marcada en el original), no va a ser entendida por el lector francés, de allí que no solo decide destacar el elemento con cursivas sino que añade una explicación que no se encuentra en el texto, es decir que no ha sido expresada por el narrador. En este caso la asunción de la enunciación se concreta a través de una explicitación que el lector asume a su vez como expresada 
por el narrador. El ethos traductor asegura pues, a la manera de un maestro, la inteligibilidad de la expresión y la consciencia del traductor como mediador cultural. Se instala pues un juego en el que el narrador es "hablado" por la instancia discursiva de la traducción, la cual asume a su vez la responsabilidad de la enunciación traductora (o re-enunciación) en otro "aquí y ahora" diferente del texto previo.

\section{Conclusión}

Hemos intentado trazar un recorrido en el que la traducción como género en sí mismo contiene numerosas aristas, desde su presencia histórica en la literatura francesa hasta su interés por ser investigada por las teorías actuales atravesadas por los estudios culturales y el Análisis del Discurso.

Hemos rastreado los indicios de la traducción como género autónomo tanto desde la historia como desde la teoría hermenéutica germano-hispana, sin olvidar el giro de la Teoría del Polisistema. Acompañando estos desarrollos, hemos considerado que las herramientas del Análisis del Discurso complementan la caracterización del texto traducido con la noción tanto de ethos como de re-enunciación y de heterolingüismo.

En este camino investigamos, con la esperanza de tomar consciencia de que lo que estamos tratando de definir, de caracterizar y de problematizar no escapa a lo que Boase-Beier (A Critical Introduction) denomina "conceptual blend": un texto (con todo lo que implica el universo del texto) que, a la manera de un unicornio, existe antes que nada para quienes lo estudiamos, pero que, para un lector incauto, simplemente no tiene entidad. Nadie dice que ha leído la obra de tal traductor pero sí de tal autor, aun si dicho autor no es exactamente él en la lengua traducida.

Las huellas de la presencia del ethos del traductor son analizables en la materialidad del texto a través del paratexto, de las notas al pie, de los glosarios, de las convenciones tipográficas, de las reformulaciones y explicitaciones, todo lo cual compone un conjunto determinante de funciones y condiciones específicas susceptibles de caracterizar el texto traducido con sus normas, su contexto de producción, su finalidad, su responsable y su destinatario, diferentes del texto original 
y por ello con posibilidades de conformar un género discursivo diferencial. Si bien la re-enunciación, con todo lo que esta noción encierra, no es un factor suficiente, sí creemos que su análisis puede contribuir a convertirla en un fenómeno necesario para la caracterización de la traducción como género en sí mismo.

\section{Ana María Gentile}

Doctora en Ciencias del Lenguaje (Universidad de Ruán, Francia, 2007) y Magíster en Ciencias del Lenguaje por la misma Universidad (2002). Es directora de proyecto en el Área de Investigación en Traductología (AIT/IdHICS, UNLP). Se desempeña como Prof. Ord. Titular de "Traducción Literaria francés/español", "Traducción científico-técnica francés/español” y de "Capacitación en Francés" (lectura de textos de especialidad) en esa misma Universidad. Es autora de numerosas ponencias en Congresos de la especialidad, así como de capítulos de libros y artículos publicados en revistas internacionales (Neologica, Arena Romanistica y Hermeneus, entre otras). En el campo editorial, es redactora en jefe de la revista internacional Synergies Argentine.

Sus áreas de investigación son la Traductología, la Didáctica del texto especializado y de la Traducción, la Lexicología, la Terminología, las relaciones entre traducción y cultura y la traducción como género discursivo. 
Ana María Gentile

\section{OBRA CITADA}

Amossy Ruth (ed.). Images de soi dans le discours. La construction de l'ethos. Ginebra: Delachaux et Niestlé, 1999.

Amossy Ruth. L'argumentation dans le discours. Discours politique, littérature, d’idées, fiction. París : Nathan, 2000.

Amossy, Ruth. "Argumentation et Analyse du Discours : perspectives théoriques et découpages disciplinaires", Argumentation et Analyse du Discours 1 (2008), Web http://aad.revues.org/index2oo.html

Amossy, Ruth. La présentation de soi. Ethos et identité verbale. París : Presses Universitaires de France, 2010.

Authier-Revuz, Jacqueline. "Hétérogénéité(s) énonciative(s)". Langages 73 (1984): 98-111.

Baker, Mona. "Corpus Linguistics and Translation Studies - Implications and Applications". Text and Technology: In Honour of John Sinclair. Eds. Mona Baker, Gill Francis y Elena Tognini-Bonelli . Amsterdam-Philadelphia: John Benjamins, 1993: 233-250.

Bajtín, Mijail. Estética de la creación verbal. Buenos Aires: Siglo xxI, 2008.

Ballard, Michel. De Cicéron à Benjamin, Traducteurs, traductions, réflexions. Lille: Presses Universitaires de Lille, 1992.

Beacco, Jean-Claude (ed.). "Les genres textuels dans l'analyse du discours : écriture légitime et communautés translangagières". Ethnolinguistique de l'écrit. Langages 105 (1992): 8-27.

Benjamin, Walter. "La tarea del traductor”. Angelus Novus. Barcelona: Edhasa, 1971.

Benveniste, Émile. Problèmes de linguistique générale. Vol. 2. París: Gallimard, 1974.

Berman, Antoine. L'épreuve de l'étranger. Culture et traduction dans l'Allemagne romantique. Col. Les Essais. París: Gallimard, 1984.

Boase-Beier, Jean. A Critical Introduction to Translation Studies. Londres, Nueva York: Continuum International Publishing Group, 2011. 
La traducción, ¿un género en sí mismo?

Brisset, Annie. Sociocritique de la traduction. Quebec : Les Éditions du Préambule, 1990.

Cassin, Barbara (dir.). Vocabulaire européen des philosophies : Dictionnaire des intraduisibles, París : Seuil, 2004.

Charaudeau, Patrick y Maingueneau, Dominique. Diccionario de análisis del discurso. Buenos Aires, Madrid: Amorrortu, 2005.

Derrida, Jacques. Le monolinguisme de l'autre. París: Galilée, 1996.

Derrida, Jacques. "What is a 'Relevant' Translation?", Critical Inquiry 27.2 (2001): 174-200.

Ducrot, Oswald. Le dire et le dit. París: Les éditions de minuit, 1984.

Foucault, Michel. L'Archéologie du savoir. París : Gallimard, 1969.

Gentile, Ana María. "Los textos jurídicos de transición: desafíos socioterminológicos y traductológicos". Introducción a la traducción jurídica y jurada. Vol. 2. Coords. Miguel Duro Moreno, Ana Belén Martínez López, Pedro San Ginés Aguilar. 3ra. Ed. Corregida, revisada, actiualizada y aumentada dedicada al Dr. Emilio Ortega Arjonilla in memoriam. Col. Interlingua. Madrid: Comares, 2020: 215-235.

Ghirimoldi, María Eugenia. "La lectura de textos traducidos: ¿ethos de autor o ethos de traductor?". Memorias ix Congreso Internacional Orbis Tertius de Teoría y Crítica Literaria, 3 al 5 de junio de 2015, Ensenada, Argentina. Dirs. Verónica Delgado, Juan Antonio Ennis, Margarita Merbilhaá y Hernán Pas. Lectores y lectura. Homenaje a Susana Zanetti. Coord. Lea E. Hafter. Actas, 2015. Ensenada: Universidad Nacional de La Platao-Facultad de Humanidades y Ciencias de la Educación-Centro de Estudios de Teoría y Crítica Literaria. 2015. Web. http:// www.memoria.fahce.unlp.edu.ar/trab_eventos/ev.8650/ev.8650.pdf

Ghirimoldi, María Eugenia. "Retos de la traducción del imaginario creole: negociar la distancia enunciativa entre texto original heterolingüe y traducción". Belas Infiéis 8 (2019): 51-70. doi:10.26512/belasinfieis.v8.n2.2019.24378

Grutman, Rainer. Des langues qui résonnent. L'hétérolinguisme au xixe siècle québécois. Montréal: Fidès-CÉTUQ, 1997. 
Even Zohar, Itamar. "La posición de la literatura traducida en el polisistema literario". Teoría de los Polisistemas. Trad. Montserrat Iglesias Santos revisada por el autor. Estudio introductorio, compilación de textos y bibliografía de Montserrat Iglesias Santos. Col. Bibliotheca Philologica, Serie Lecturas. Madrid: Arco, 1999: 223-231.

Hermans, Theo (ed). The Manipulation of Literature. Londres y Nueva York: Routledge, 1985 .

Maingueneau, Dominique. Genèses du discours. París: Pierre Mardaga, 1984.

Maingueneau, Dominique. "Ethos, scénographie, incorporation”. Images de soi dans le discours. La construction de l'ethos, Lausanne. París: Delauchaux et Niestlé S.A., 1999: 75-100.

Maingueneau, Dominique. Analyser les textes de communication. 2e ed. París: Armand Colin, 2009.

Masson, Jean-Yves. "Traductologie et histoire des traductions : le tournant historique de la traductologie et le tournant traductologiqiue de l'Histoire". Conferencia inaugural del 1er Congreso Mundial de Traductología, Universidad de Nanterra, París. Web. https://crea.parisnanterre.fr/1er-congres-mondial-de-traductologie-761385.kjsp

Pym, Anthony. Teorías contemporáneas de la traducción, materiales para un curso universitario. 2 da edición. Intercultural Studies Group. Tarragona, España: URV, 2016. Web. http://isg.urv.es/publicity/isg/publications/2011_teorias/pym_teorias_traduccion_web.pdf

Ortega y Gasset, José. “Miseria y esplendor de la traducción”. Obras Completas V. 1a edición. Madrid: Revista de Occidente-Alianza Editorial, 1983: 431-452.

Risterucci-Roudnicky, Danièle. Introduction à l'analyse des oeuvres traduites. París: Armand Colin, 2008.

Schleiermacher, Friedrich. Sobre los diferentes métodos de traducir. Trad. y coments. de Valentín García Yebra. Edición bilingüe. Gredos: Madrid, 2000. 
Spoturno, María Laura. "The Presence and Image of the Translator in Narrative Discourse: towards a Definition of the Translator's Ethos”. Moderna Språk 1 (2017): 173-196.

Spoturno, María Laura. "El retrabajo del ethos en el discurso autotraducido. El caso de Rosario Ferré”. Hermeneus 21 (2019): 323-354.

Suchet, Myriam. "La traduction, une éthique de la ré-énonciation”. Nouvelle revue d'esthétique 1.3 (2009) 31-35.

Suchet, Myriam. "Voice, Tone and Ethos: A Portrait of the Translator as a Spokesperson". La traduction des voix intra-textuelles/Intertextual Voices in Translation. Eds. Kristiina Taivalkoski-Shilov and Myriam Suchet. Montreal: Éditions québécoises de l'oeuvre, 2013: 159-184. https://pdfs.semanticscholar. org/of3e/6c99ffacoc9bboabao236f203ede7982569f.pdf

Suchet, Myriam. L'Imaginaire hétérolingue. Ce que nous apprennent les textes à la croisée des langues. París: Classiques Garnier, 2014.

Toury, Gideon. Descriptive Translation Studies - And Beyond. Ámsterdam y Filadelfia: John Benjamins, 1995.

Willson, Patricia. La constelación del Sur. Buenos Aires: Siglo XXI, 2004.

Woether, Frédérique. L'Ėthos aristotélicien: genèse d'une notion rhétorique. París: Vrin, 2007. 